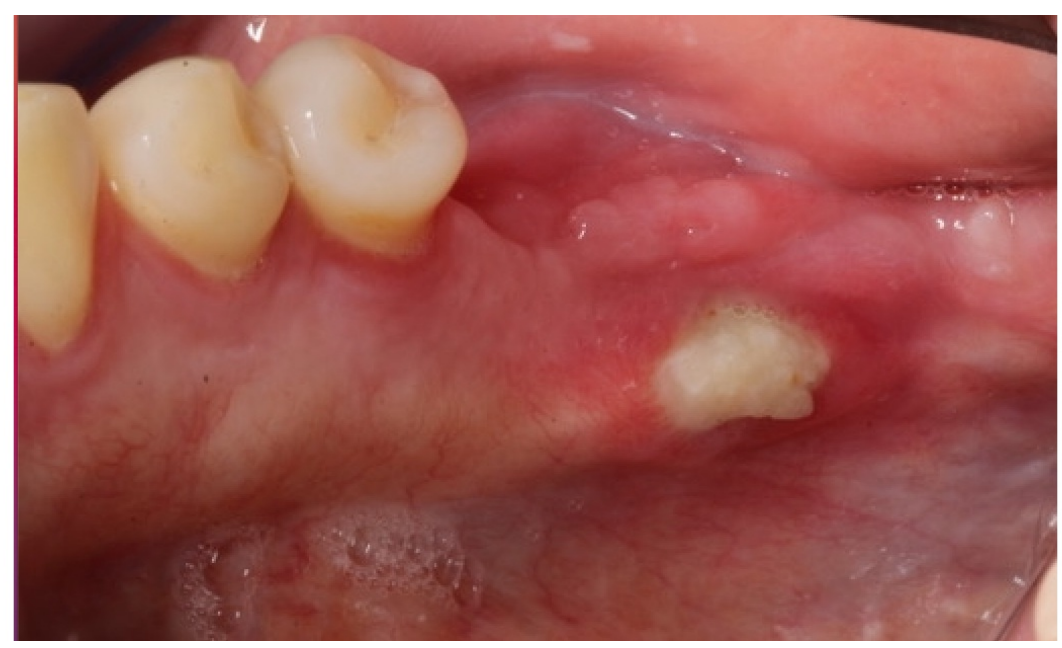

\title{
OSTEONECROSIS OF JAW (ONJ) IN PATIENTS WITH OSTEOPOROSIS AND OTHER NON-MALIGNANT DISEASES IN PIEDMONT AND AOSTA VALLEY 2004-2018
} \author{
Fusco $^{6}$

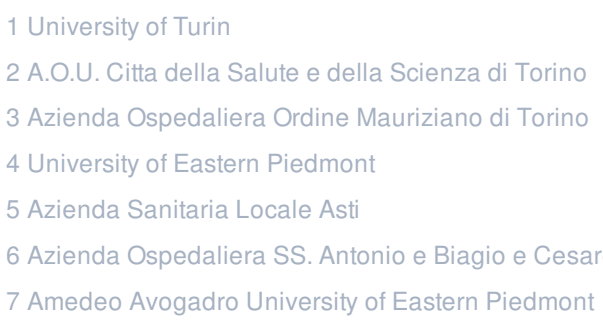

Alessio Gambino ${ }^{1}$, MARCO CABRAS ${ }^{1}$, Francesco Maria Erovigni ${ }^{1}$, Alessandro Dell'Acqua ${ }^{2}$, FRANCESCO DELLA FERRERA $^{3}$, Paolo Appendino ${ }^{3}$, Stefano Franchi ${ }^{4}$, Majlinda Caka ${ }^{5}$, Antonella Fasciolo ${ }^{6}$, Mario Migliario ${ }^{7}$, Guglielmo Ramieri ${ }^{1}$, Paolo Garzino Demo ${ }^{1}$, Monica Pentenero ${ }^{1}$, lia giordano ${ }^{1}$, Massimo Carossa ${ }^{1}$, Paolo Giacomo Arduino ${ }^{1}$, Vittorio

Funding: The author(s) received no specific funding for this work.

Potential competing interests: The author(s) declared that no potential competing interests exist.

\section{Abstract}

Osteonecrosis of the Jaws (ONJ) related to bisphosphonates (BPs) or denosumab is a concern for osteoporosis and Rheumatoid Arthritis (RA) patients. In a context of increasing prescription rate of BPs and denosumab amid those patients in the last decade, incidence and/or prevalence of ONJ in these categories of patients remain uncertain, with lack of solid epidemiologic data from observational studies. As an almost unique experience, the present work provides a multicenter, retrospective overview of ONJ in non malignant disease patients over a timespan of 15 years (Jan $1^{\text {st }}$ 2004 - Dec $31^{\text {st }}, 2018$ ) from the oral care centers of Piedmont and Valle d'Aosta. In detail, data from 165 patients (8 M, $157 \mathrm{~F})$ were acquired. Mean age was of $74(38-91)$ years, with more than half $(64.2 \%)$ of patients diagnosed with ONJ 
between 70 and 89 years. Underlying diseases were osteoporosis (80\%), RA (5.4\%), osteoporosis and RA (3.6\%), other bone disorders (11\%). Treatment administered were alendronate (51.8\%), ibandronate (12.3\%), zoledronic acid (6.8\%), others (29.1\%). Concerning the latter, since 2014, 7 cases were related to denosumab (60 mg every 6 months), of which 5 to denosumab alone, 2 in patients switched from BPs to denosumab. Sites of ONJ were mandible (67\%), maxilla (25\%), mandible and maxilla (7\%). Finally, an overall rise of ONJ occurrence was observed, with a median of 7 (2-13) yearly ONJ cases in the 2004-2010 period, gone up to 11.5 (6-24) yearly ONJ events in the 2011-2018 period. Such worrisome trend underlines the importance of eventual prevention measures and recurring assessment of oral health to reduce ONJ risk in non-malignant disease patients.

Background: Despite Osteonecrosis of Jaws (ONJ) related to bisphosphonates (BPs) or denosumab is mostly associated to cancer and myeloma population ([1]), it should be also considered a potential adverse effect among the increasing population of patients affected by bone metabolic disorders or non-metastatic cancer, in need for BPs (oral alendronate, oral risedronate, oral/IV ibandronate, IV zoledronic acid) or subcutaneous denosumab (60 mg every 6 months) to prevent fractures.

With scattered epidemiologic data, incidence and prevalence of ONJ among this population is far from being assessed, ranging from an occurrence reported as "very rare" ( ${ }^{[2]}$ ) to an observation even more frequent than ONJ in metastatic cancer patients, in absolute terms ${ }^{[3]}$. . Aim of the present work was to retrospectively describe number of registered cases and main features of ONJ cases observed at main hospital oral care centers, in a timespan of 15 years (2004-2018) in patients with non-malignant diseases (primary osteoporosis, secondary osteoporosis or osteopenia, Rheumatoid Arthritis and other rheumatologic diseases) in Piedmont and Valle d'Aosta territory (4.4 million inhabitants). Materials and Methods: Data were retrospectively collected from Oral Medicine, Oral Surgery and Oral Maxillofacial Surgery Units, from January 1st 2004 to 31st December 2018. The main parameters collected were: underlying bone metabolic disorder (as registered in dental care charts); treatment protocol - either bisphosphonates alone, or denosumab, or bisphosphonate(s)/denosumab sequence; age of patients at year of $\mathrm{ONJ}$ diagnosis; mandibular and/or maxillary site of ONJ onset.

Results: Over the 2004-2018 timespan, data from 165 patients were acquired. Of these, 95\% were females. Mean age was of 74 (38-91) years, with more than half $(64.2 \%)$ of patients being diagnosed with ONJ between 70 and 89 years.

Underlying disease was reported as osteoporosis (80\%), rheumatoid arthritis $(5.4 \%)$, osteoporosis and rheumatoid arthritis (3.6\%), or other bone disorders (11\%). The bisphosphonates most commonly administered were alendronate (51.8\%), ibandronate (12.3\%), zoledronic acid (6.8\%). Since 2014, 7 cases were related to denosumab (60 mg every 6 months), either alone (5 cases) or as part of a sequence with alendronate or ibandronate ( 2 cases, respectively). The remaining cases were related to clodronate, neridronate, pamidronate, and risedronate, either alone or as sequences with other drugs. Sites of ONJ were mandible (67\%), maxilla (25\%), with maxillary and mandibular ONJ encountered in the remaining 7\%. Throughout the 15 years period of investigation, an increasing trend emerged, with a median of 7 (range 213) yearly ONJ cases in the 2004-2010 period, increased to 11.5 (range 6-24) yearly ONJ events in the 2011-2018 period. Conclusions: ONJ appeared to be increasing in frequency over the last 15 years among patients affected by bone metabolic disorders, occurring so far with predilection for mandible sites, in elderly (>70 years) and female patients, mostly 
during/after bisphosphonate(s) treatment. ONJ cases solely related to denosumab were so far anecdotal, although such frequency might arise in the next years, due to an increasing propensity for prescription of denosumab as first-line treatment against bone metabolic disorders, as well as a "switch" drug from protracted bisphosphonates regimens ([4]) Further recollection of clinical data - concerning comorbidities, oral health risk factors, differential radiological features and surgical outcomes - should be pursued, to clarify the main characteristics of these novel clusters of patients, emerging out of the wide population of osteoporotic elders.

\section{References}

1. 'Giuseppina Campisi, Stefano Fedele, Vittorio Fusco, Giuseppe Pizzo, et al. (2014). Epidemiology, clinical manifestations, risk reduction and treatment strategies of jaw osteonecrosis in cancer patients exposed to antiresorptive agents. Future Oncology, vol. 10 (2), 257-275. doi:10.2217/fon.13.211.

2. 'Robert A Adler, Ghada El-Hajj Fuleihan, Douglas C Bauer, Pauline M Camacho, et al. (2016). Managing Osteoporosis in Patients on Long-Term Bisphosphonate Treatment: Report of a Task Force of the American Society for Bone and Mineral Research. J Bone Miner Res, vol. 31 (1), 16-35. doi:10.1002/jbmr.2708.

3. `Jeong Keun Lee et al.. (2013). Bisphosphonates-related osteonecrosis of the jaw in Korea: a preliminary report. J Korean Assoc Oral Maxillofac Surg ..

4. ^Noam Yarom, Towy Sorel Lazarovici, Sara Whitefield, Tal Weissman, et al. (2018). Rapid onset of osteonecrosis of the jaw in patients switching from bisphosphonates to denosumab. Oral Surgery, Oral Medicine, Oral Pathology and Oral Radiology, vol. 125 (1), 27-30. doi:10.1016/j.0ooo.2017.09.014. 